\title{
Bioinformatics analysis and experimental validation of differentially expressed genes in mouse articular chondrocytes treated with IL-1ß using microarray data
}

\author{
FAN LIANG，LE PENG，YONG-GANG MA，WEI HU，WEI-BING ZHANG，MING DENG and YA-MING LI \\ Department of Orthopedics, Renmin Hospital of Wuhan University, Wuhan, Hubei 430060, P.R. China
}

Received July 14, 2020; Accepted February 2, 2021

DOI: $10.3892 /$ etm.2021.10928

\begin{abstract}
Osteoarthritis (OA) is the most prevalent chronic degenerative disease that affects the health of the elderly. The present study aimed to identify significant genes involved in OA via bioinformatics analysis. A gene expression dataset (GSE104793) was downloaded from the Gene Expression Omnibus. Bioinformatics analysis was then performed in order to identify differentially expressed genes (DEGs) between untreated chondrocytes and chondrocytes cultured with interleukin-1 $\beta$ (IL-1 $\beta$ ) for $24 \mathrm{~h}$. Gene Ontology (GO) and Kyoto Encyclopedia of Genes and Genomes (KEGG) pathway enrichment analyses were performed using Metascape. A protein-protein interaction network of DEGs was constructed using the Search Tool for the Retrieval of Interacting Genes. Gene set enrichment analysis (GSEA) was performed using GSEA software. Furthermore, chondrocytes were extracted and treated with IL-1 $\beta$ (10 $\mathrm{ng} / \mathrm{ml})$ for $24 \mathrm{~h}$, and reverse-transcription quantitative PCR was used to confirm differential expression of hub genes. Patient samples were also collected to verify the bioinformatic analysis results. Based on the cut-off criteria used for determination of the DEGs, a total of 844 DEGs, including 498 upregulated and 346 downregulated DEGs, were identified. The DEGs were mainly enriched in the GO terms and KEGG pathways 'inflammatory response', 'negative regulation of cell proliferation', 'ossification', 'taxis', 'blood vessel morphogenesis', 'extracellular structure organization', 'mitotic cell cycle process' and 'TNF
\end{abstract}

Correspondence to: Professor Ya-Ming Li, Department of Orthopedics, Renmin Hospital of Wuhan University, 238 Jiefang Road, Wuchang, Wuhan, Hubei 430060, P.R. China

E-mail: 1i99770@163.com

Abbreviations: OA, osteoarthritis; GEO, Gene Expression Omnibus; GO, Gene Ontology; KEGG, Kyoto Encyclopedia of Genes and Genomes; PPI, protein-protein interaction; STRING, Search Tool for the Retrieval of Interacting Genes; GSEA, Gene set enrichment analysis; IL- $1 \beta$, interleukin- $1 \beta$; DEGs, differentially expressed genes; MCODE, Molecular Complex Detection

Key words: osteoarthritis, bioinformatics analysis, differentially expressed genes signaling pathway'. The majority of the PCR results, namely the differential expression of kininogen 2, complement C3, cyclin B1, cell division cycle 20, cyclin A2, 1-phosphatidylinositol 4-kinase, BUB1 mitotic checkpoint serine/threonine kinase, kinesin family member 11, cyclin B2 and BUB1 mitotic checkpoint serine/threonine kinase B were consistent with the bioinformatics results. Collectively, the present observations provided a regulation network of IL- $1 \beta$-stimulated chondrocytes, which may provide potential targets of OA therapy.

\section{Introduction}

Osteoarthritis (OA) is the most common age-associated degenerative disease, which causes pain, stiffness and decreased function of the joints (1-3). With the increasing aging of the population, the incidence of $\mathrm{OA}$ is on the rise and affects $>250$ million individuals worldwide $(4,5)$. Thus far, there is no effective treatment available for OA (6). Non-steroidal anti-inflammatory drugs are approved for the symptomatic treatment of OA only (7). These drugs are only symptomatic treatments and do not delay the progression of OA (8). Therefore, understanding the mechanisms underlying the promotion of $\mathrm{OA}$ is essential for developing effective therapies for OA.

The onset of OA is dependent on TNF- $\alpha$, interleukin (IL)-1 $\beta$ and IL-12 (9). IL-1 $\beta$ is considered the initiator of the acute inflammatory response in OA (10) and has been demonstrated to be implicated in inducing chondrocyte apoptosis and degradation; thus, it may be used to generate an in vitro cellular model of OA (11).

Apoptosis is known to be important in the pathophysiological processes of OA and IL-1 $\beta$ has been reported to accelerate aging and increase the apoptotic index of chondrocytes (12). It is known that loss of chondrocytes induced by IL-1 $\beta$ decreases the production of the chondrocyte matrix and increases cartilage damage (13). However, the changes in genes and the specific regulatory network during IL-1 $\beta$-treated chondrocytes remain poorly understood.

It is therefore important to understand the gene changes in chondrocytes caused by IL-1 $\beta$. With the development of high-throughput sequencing technology and bioinformatics, numerous mRNAs have been identified. Microarray techniques and bioinformatics analyses have been extensively used to screen for differences at the genomic level involved 
in IL-1 $\beta$-stimulated chondrocyte apoptosis, enabling the identification of differentially expressed genes (DEGs) and the potential biological processes associated with OA progression.

In the present study, a microarray dataset was downloaded from the Gene Expression Omnibus (GEO) database and analyzed. Furthermore, potential Gene Ontology (GO) functional terms and pathways of these DEGs were analyzed. A protein-protein interaction (PPI) network was then established in order to elucidate the hub genes that are involved in OA.

\section{Materials and methods}

Gene expression microarray data. Datasets comparing chondrocytes that had been treated with either IL-1 $\beta$ or placebo were selected. Search terms were as follows: Chondrocytes and IL-1 $\beta$. The gene expression profile from the dataset GSE104793 was downloaded from GEO (www.ncbi.nlm.nih. gov/geo/). GSE104793 was based on the Affymetrix Mouse Gene 2.0 ST Array [transcript (gene) version] platform. The GSE104793 dataset contained six samples, including three untreated mouse articular chondrocyte samples and three IL-1 $\beta$-treated primary mouse articular chondrocyte samples. The dataset GSE74220 was also downloaded from GEO. The GSE74220 dataset contained six samples, including three untreated human articular chondrocytes samples and three IL-1 $\beta$-treated primary human articular chondrocyte samples.

Differential expression analyses. Before the data analysis, normalization was performed to eliminate any discrepancies based on non-conforming units. Raw data were processed via robust multi-array average algorithm methods, as previously described (14). The Limma package in R software was used to select DEGs (14). To screen for DEGs, the cut-off criteria were set as fold change $(\mathrm{FC})>2(\operatorname{lLog} \mathrm{FCl}>1)$ and $\mathrm{P}<0.05$. Volcano plots were generated using the R package ggplot (15). A heatmap was drawn using the heatmap package of the $\mathrm{R}$ software (version 3.5.0).

GO and Kyoto Encyclopedia of Genes and Genomes (KEGG) analysis of DEGs. Metascape is an online gene enrichment tool used to extract comprehensive biological information associated with gene lists. GO and KEGG enrichment analyses were performed with the Metascape online tool (http://metascape. org/gp/index.html\#/main/step1) (16). GO terms, including those in the categories biological process, cellular component and molecular function, were determined. Furthermore, Gene Set Enrichment Analysis (GSEA) (http://software.broadinstitute.org/gsea) was performed.

PPI. Numerous DEGs are involved in the progress of IL-1 $\beta$-induced chondrocytes and the association between these DEGs was determined using the Search Tool for the Retrieval of Interacting Genes (STRING) database (https://string-db. org/). A minimum required interaction score $>0.9$ was set as the criterion. Networks were visualized using Cytoscape (version 3.7.1) (17). Subsequently, a module analysis of the network was performed using the plugin Molecular Complex Detection (MCODE). The MCODE criteria for selection
Table I. General characteristics of the patients of the present study.

\begin{tabular}{lccc}
\hline Parameter & OA $(\mathrm{n}=20)$ & Control $(\mathrm{n}=20)$ & P-value \\
\hline Age $($ years $)$ & $67.56 \pm 5.69$ & $65.19 \pm 6.53$ & 0.518 \\
BMI $\left(\mathrm{kg} / \mathrm{m}^{2}\right)$ & $24.53 \pm 4.78$ & $22.64 \pm 3.36$ & 0.049 \\
Body height $(\mathrm{m})$ & $162.45 \pm 7.31$ & $161.86 \pm 6.47$ & 0.502 \\
Body weight $(\mathrm{kg})$ & $64.29 \pm 10.91$ & $59.44 \pm 10.24$ & 0.731 \\
Sex & & & 0.429 \\
Male & 5 & 3 & \\
Female & 15 & 17 & \\
\hline
\end{tabular}

OA, osteoarthritis; BMI, body mass index.

were as follows: Degree cut-off $\geq 10$, node score cut-off $\geq 0.4$, $\mathrm{K}$-core $\geq 4$ and max depth $=100$.

Cell culture. Primary chondrocytes were isolated from mouse femoral condyles and tibial plateau cartilage tissue as previously described (18). A total of $10 \mathrm{C} 57 \mathrm{BL} / 6 \mathrm{~J}$ male mouse pups (Beijing Vital Laboratory Animal Technology Co., Ltd; body weight, 2.5-3.0 g; age, 5-7 days) were housed under standard laboratory conditions $\left(22 \pm 2^{\circ} \mathrm{C}, 12\right.$-h light/dark cycle) with food and water available ad libitum. Cervical dislocation euthanasia of all mouse pups was performed after pentobarbital sodium anesthesia $(40 \mathrm{mg} / \mathrm{kg}$; I.V.). In brief, the obtained cartilage fragments were cut into small pieces and washed with PBS. Subsequently, cartilage pieces were digested with $0.2 \%(w / v)$ collagenase type II (Sigma Aldrich; Merck KGaA) for $4 \mathrm{~h}$ at $37^{\circ} \mathrm{C}$. Next, 200 mesh-filtrating screens were used to remove large fragments. Collected chondrocytes were cultured with Dulbecco's modified Eagle's medium (Gibco; Thermo Fisher Scientific, Inc.) supplemented with $25 \%$ fetal bovine serum (BD Biosciences). Cells at the second passage were used for the subsequent experiments. Chondrocytes were stimulated with IL-1 $\beta(10 \mathrm{ng} / \mathrm{ml})$ for $24 \mathrm{~h}$ as previously described (19). The present study was approved by The Ethics Committee of Renmin Hospital of Wuhan University (approval no. 2019-YLS-0125, Wuhan, China).

Apoptosisassay.TheapoptoticchondrocytesintheIL-1 $\beta$-treated group were identified using an Annexin V-FITC/propidium iodide (PI) double staining kit (cat. no. 556547; BD Biosciences) according to the manufacturer's protocol. In brief, IL-1 $\beta$-treated chondrocytes $\left(5 \times 10^{6}\right.$ cells/tube) were collected in a centrifuge tube. Subsequently, working solution $(5 \mu 1$ of FITC Annexin V and $1 \mu \mathrm{l}$ of $50 \mu \mathrm{g} / \mathrm{ml} \mathrm{PI}$ ) was added, followed by incubation at room temperature in the dark for $15 \mathrm{~min}$. Analysis of apoptosis was performed using flow cytometry (BD Biosciences).

Human cartilage. Human cartilage samples were collected from the Orthopedics Department of Renmin Hospital of Wuhan University (Wuhan, China). The present study was approved by the Ethics Committee of Renmin Hospital of Wuhan University (Wuhan, China). Written informed consent was obtained from all subjects. 
Table II. Top 20 upregulated differentially expressed genes in interleukin-1 $\beta$-treated chondrocytes compared with those of the control group.

\begin{tabular}{|c|c|c|c|c|c|c|}
\hline Gene & $\operatorname{logFC}$ & AveExpr & $\mathrm{t}$ & P-value & adj.P.Val & $\mathrm{B}$ \\
\hline Serum amyloid A2 & 5.9163 & 6.5406 & 73.1477 & $2.46 \times 10^{-13}$ & $6.06 \mathrm{E}-09$ & 18.6193 \\
\hline $\mathrm{C}-\mathrm{X}-\mathrm{C}$ motif chemokine ligand 5 & 6.5031 & 8.6703 & 64.0081 & $7.74 \times 10^{-13}$ & $9.54 \mathrm{E}-09$ & 18.1034 \\
\hline Matrix metallopeptidase 3 & 7.2795 & 8.1056 & 59.9409 & $1.36 \times 10^{-12}$ & $1.12 \mathrm{E}-08$ & 17.8193 \\
\hline $\mathrm{C}-\mathrm{X}-\mathrm{C}$ motif chemokine ligand 1 & 5.6297 & 8.4909 & 56.7565 & $2.18 \times 10^{-12}$ & $1.13 \mathrm{E}-08$ & 17.5675 \\
\hline $\mathrm{C}-\mathrm{C}$ motif chemokine ligand 7 & 5.1185 & 7.8909 & 56.4355 & $2.29 \times 10^{-12}$ & $1.13 \mathrm{E}-08$ & 17.5405 \\
\hline Nitric oxide synthase 2 & 6.2695 & 7.4200 & 54.3523 & $3.16 \times 10^{-12}$ & $1.30 \mathrm{E}-08$ & 17.3578 \\
\hline Matrix metallopeptidase 13 & 4.4475 & 10.2420 & 53.0409 & $3.89 \times 10^{-12}$ & $1.37 \mathrm{E}-08$ & 17.2355 \\
\hline Vanin 1 & 5.9276 & 8.1563 & 48.6124 & $8.23 \times 10^{-12}$ & 2.54E-08 & 16.7762 \\
\hline $\mathrm{C}-\mathrm{C}$ motif chemokine ligand 2 & 5.9043 & 7.8825 & 45.4236 & $1.47 \times 10^{-11}$ & 4.03E-08 & 16.3943 \\
\hline Haptoglobin & 6.0710 & 8.0656 & 44.0942 & $1.90 \times 10^{-11}$ & 4.69E-08 & 16.2205 \\
\hline Serum amyloid A1 & 5.6294 & 5.3605 & 41.9865 & $2.89 \times 10^{-11}$ & $6.48 \mathrm{E}-08$ & 15.9255 \\
\hline Vanin 3 & 4.2944 & 6.2530 & 41.0399 & $3.52 \times 10^{-11}$ & 7.23E-08 & 15.7845 \\
\hline Serpin family G member 1 & 4.9816 & 6.8803 & 37.9014 & $6.96 \times 10^{-11}$ & $1.32 \mathrm{E}-07$ & 15.2758 \\
\hline SLIT and NTRK like family member 6 & 3.8563 & 6.9691 & 37.0835 & $8.39 \times 10^{-11}$ & $1.48 \mathrm{E}-07$ & 15.1317 \\
\hline $\mathrm{C}-\mathrm{X}-\mathrm{C}$ motif chemokine ligand 3 & 7.9719 & 6.9130 & 35.7119 & $1.16 \times 10^{-10}$ & $1.90 \mathrm{E}-07$ & 14.8786 \\
\hline Serum amyloid A4, constitutive & 4.2909 & 5.7558 & 34.6319 & $1.51 \times 10^{-10}$ & 2.17E-07 & 14.6682 \\
\hline NFKB inhibitor zeta & 3.2694 & 7.6966 & 34.6222 & $1.51 \times 10^{-10}$ & 2.17E-07 & 14.6663 \\
\hline Cholesterol 25-hydroxylase & 3.0854 & 5.8962 & 34.4341 & $1.58 \times 10^{-10}$ & 2.17E-07 & 14.6286 \\
\hline Adrenomedullin & 3.7689 & 8.3151 & 32.1049 & $2.88 \times 10^{-10}$ & 3.73E-07 & 14.1347 \\
\hline Solute carrier family 39 member 8 & 3.4395 & 8.4606 & 31.7193 & $3.19 \times 10^{-10}$ & 3.93E-07 & 14.0478 \\
\hline
\end{tabular}

FC, fold change; AveExpr, average expression; adj.P.Val, adjusted P-value.

A
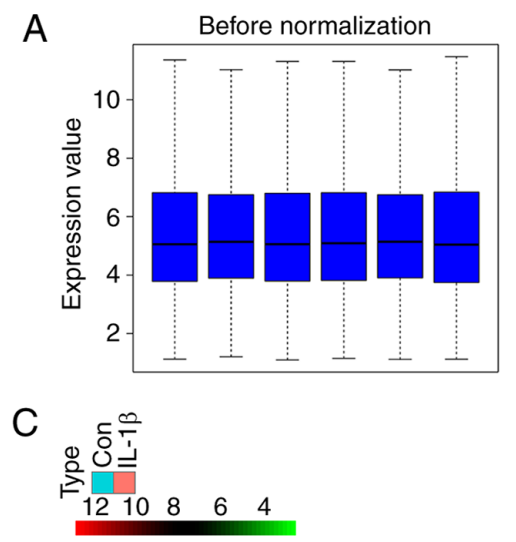
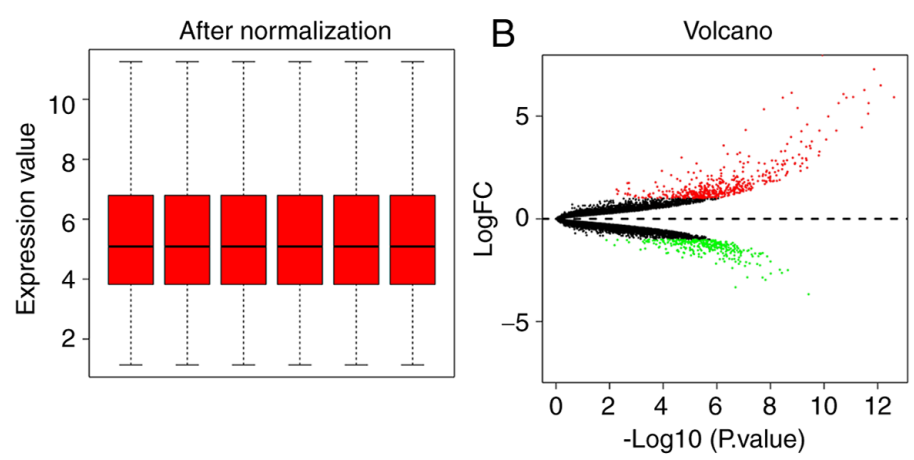

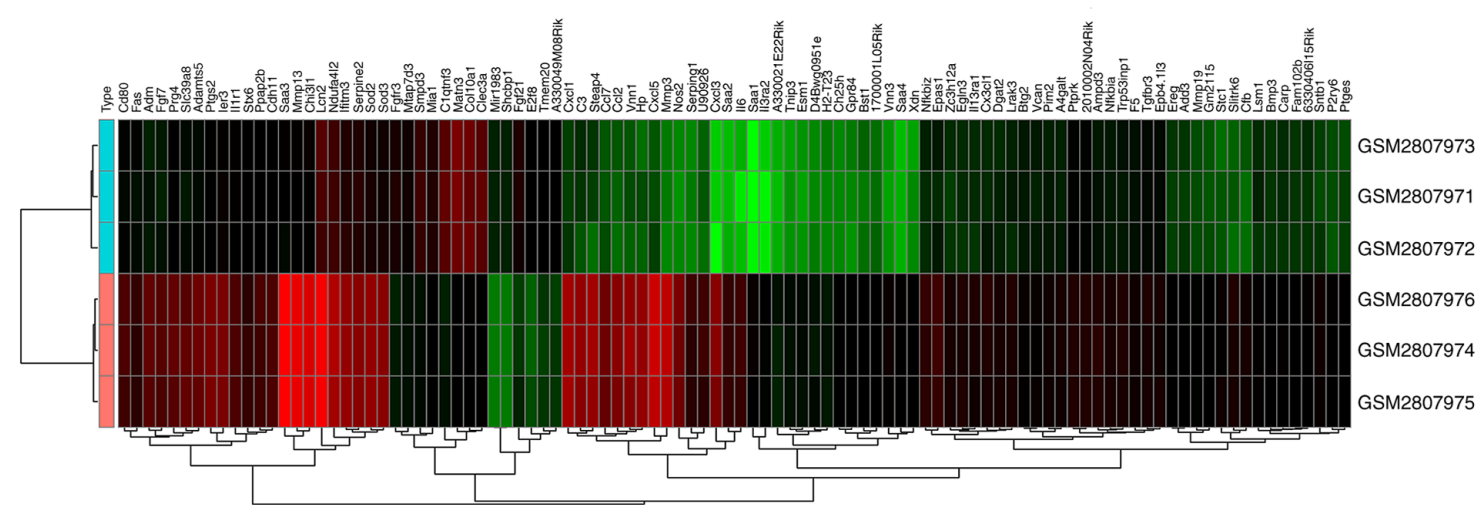

Figure 1. DEG analysis between IL-1 $\beta$-treated chondrocytes and normal chondrocytes. (A) Data prior to and after normalization. (B) Volcano plot of 844 DEGs. Red dots indicate upregulated genes and green dots represent downregulated genes, while black dots indicate genes that were not significantly changed. (C) Heatmap of the top 100 DEGs with a fold change of $>2$. Green indicates high expression and red indicates low expression. DEGs, differentially expressed genes; IL, interleukin; con, control; FC, fold change. 
Table III. Top 20 downregulated differentially expressed genes in interleukin-1 $\beta$-treated chondrocytes compared with that of the control group.

\begin{tabular}{lcccccc}
\hline Gene & logFC & AveExpr & $\mathrm{t}$ & P-value & adj.P.Val & B \\
\hline C1q and TNF related 3 & -3.6690 & 8.3401 & -31.0716 & $3.81 \times 10^{-10}$ & $4.27 \mathrm{E}-07$ & 13.8982 \\
Collagen type X alpha 1 chain & -2.5009 & 9.1546 & -25.2285 & $2.25 \times 10^{-9}$ & $1.34 \mathrm{E}-06$ & 12.3160 \\
Sphingomyelin phosphodiesterase 3 & -2.6276 & 8.1092 & -23.7519 & $3.76 \times 10^{-9}$ & $1.68 \mathrm{E}-06$ & 11.8366 \\
Fibroblast growth factor receptor 3 & -2.4767 & 7.6773 & -23.2467 & $4.51 \times 10^{-9}$ & $1.92 \mathrm{E}-06$ & 11.6638 \\
E2F transcription factor 8 & -2.6635 & 6.4849 & -21.2008 & $9.85 \times 10^{-9}$ & $3.39 \mathrm{E}-06$ & 10.9127 \\
Solute carrier family 35 member G1 & -2.0720 & 6.8393 & -21.1527 & $1.00 \times 10^{-8}$ & $3.39 \mathrm{E}-06$ & 10.8940 \\
MAP7 domain containing 3 & -1.8313 & 7.6434 & -20.4788 & $1.32 \times 10^{-8}$ & $4.07 \mathrm{E}-06$ & 10.6263 \\
Fibroblast growth factor 21 & -2.9702 & 7.3424 & -20.1300 & $1.53 \times 10^{-8}$ & $4.37 \mathrm{E}-06$ & 10.4835 \\
Melanoma inhibitory activity 1 & -1.6634 & 8.1435 & -19.5582 & $1.95 \times 10^{-8}$ & $5.11 \mathrm{E}-06$ & 10.2431 \\
Matrilin 3 & -2.8530 & 9.1627 & -19.5269 & $1.97 \times 10^{-8}$ & $5.12 \mathrm{E}-06$ & 10.2296 \\
mir1983 & -1.7090 & 5.4264 & -19.1513 & $2.33 \times 10^{-8}$ & $5.94 \mathrm{E}-06$ & 10.0668 \\
Noncompact myelin associated protein & -1.6768 & 6.6249 & -19.1406 & $2.34 \times 10^{-8}$ & $5.94 \mathrm{E}-06$ & 10.0621 \\
C-type lectin domain family 3 member A & -2.2555 & 9.0487 & -19.0299 & $2.45 \times 10^{-8}$ & $6.17 \mathrm{E}-06$ & 10.0134 \\
SHC binding and spindle associated 1 & -1.6970 & 5.4181 & -18.8890 & $2.61 \times 10^{-8}$ & $6.50 \mathrm{E}-06$ & 9.9509 \\
Cytochrome b5 reductase 2 & -1.8194 & 6.9376 & -18.7210 & $2.82 \times 10^{-8}$ & $6.80 \mathrm{E}-06$ & 9.8757 \\
Solute carrier family 38 member 3 & -2.1951 & 8.1691 & -18.4921 & $3.12 \times 10^{-8}$ & $7.33 \mathrm{E}-06$ & 9.7720 \\
Kinesin family member 11 & -1.6817 & 6.2994 & -18.0989 & $3.74 \times 10^{-8}$ & $8.70 \mathrm{E}-06$ & 9.5903 \\
Family with sequence similarity 180 & -1.8312 & 6.7883 & -17.6541 & $4.61 \times 10^{-8}$ & $1.05 \mathrm{E}-05$ & 9.3793 \\
member A & & & & & & \\
Myelin protein zero like 2 & -1.7372 & 6.1523 & -17.4783 & $5.02 \times 10^{-8}$ & $1.10 \mathrm{E}-05$ & 9.2942 \\
mir323 & -1.4407 & 5.4136 & -17.4224 & $5.16 \times 10^{-8}$ & $1.12 \mathrm{E}-05$ & 9.2670 \\
\hline
\end{tabular}

FC, fold change; AveExpr, average expression; adj.P.Val, adjusted P-value. miR-, microRNA.

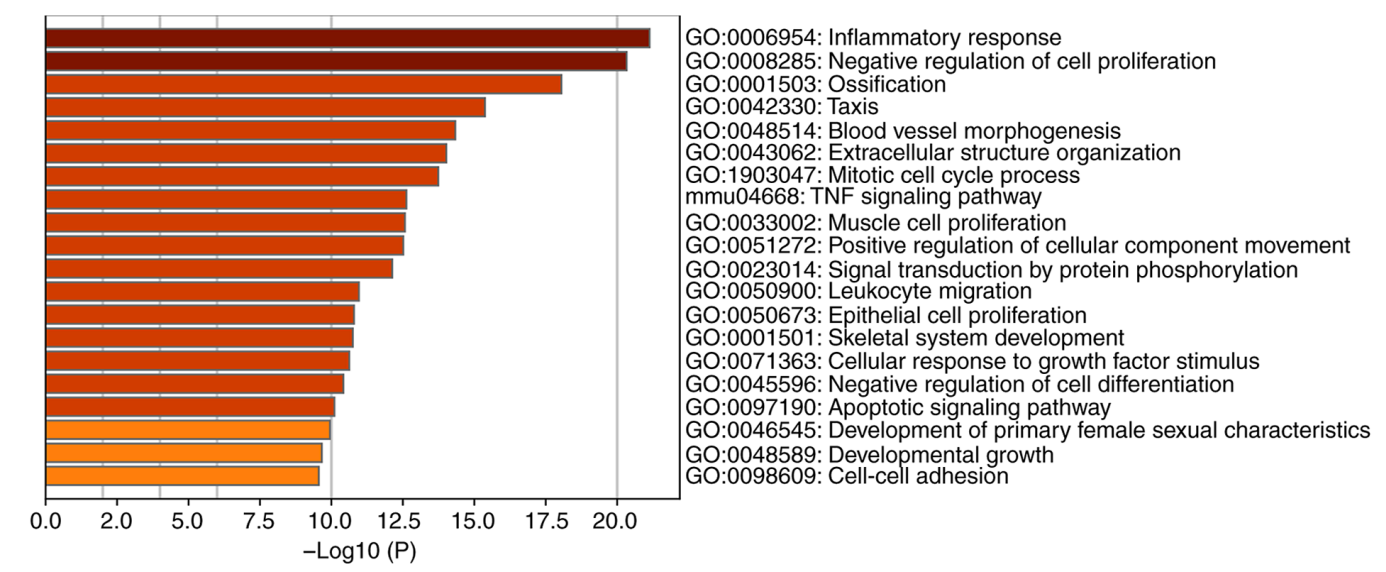

Figure 2. Bar graph of enriched terms across input gene lists, colored by P-values; a darker color indicates a greater-log10 (P-value). GO, Gene Ontology. mmu, mus musculus.

Control cartilage was harvested from patients with femoral neck fractures without any history of OA at the time-point of total hip arthroplasty $(\mathrm{n}=20)$, whereas the pathological cartilage was acquired from patients with end-stage symptomatic hip-OA at the time-point of total hip replacement surgery $(n=20)$. The clinical characteristics of the patients are presented in Table I.

$P C R$. Total RNA from the chondrocytes was extracted using TRIzol $^{\circledR}$ (Invitrogen; Thermo Fisher Scientific, Inc.) and was preserved at $-80^{\circ} \mathrm{C}$. The concentration of total RNA was quantified using a NanoDrop Lite spectrophotometer (Thermo Fisher Scientific, Inc.). Complementary (c)DNA was synthesized from $1 \mu \mathrm{g}$ total RNA using a BeyoRT First-Strand cDNA synthesis kit (Beyotime Institute of Biotechnology). Reverse transcription-quantitative PCR (RT-qPCR) was performed using a BeyoFast Probe One-Step RT-qPCR kit (Beyotime Institute of Biotechnology). In the detection system (LineGene 9600 Plus; SIA Biosan) the following thermocycling conditions were used: $95^{\circ} \mathrm{C}$ for $10 \mathrm{~min}$, followed by 45 cycles of 
$95^{\circ} \mathrm{C}$ for $30 \mathrm{sec}, 60^{\circ} \mathrm{C}$ for $30 \mathrm{sec}$ and $72^{\circ} \mathrm{C}$ for $30 \mathrm{~min}(20)$. The primers for each gene were as follows: Kininogen 2 (KNG2) forward, 5'-TGGCCTCGAGATGTGCTTCAG-3' and reverse, 5'-TCTCCTTGGCGGCCGCACTTCCTTC-3'; cyclin B1 (CCNB1) forward, 5'-AACACGCTGCCTGTCTAC ACT-3' and reverse, 5'-CAGTGCAGGGTCCGAGGT-3'; cell division cycle 20 (CDC20) forward, 5'-ACACCCGTGAGA GAGACTTG-3' and reverse, 5'-AAGTCAGTCGGGAAG GAAGG-3'; cyclin A2 (CCNA2) forward, 5'-GACCCCTTT ACTCTGACCCC-3' and reverse, 5'-AGGCTCCAGTGA ATTCGGAA-3'; 1-phosphatidylinositol 4-kinase (PIK1) forward, 5'-ACAGATGAAGTGCTCCTTCCA-3' and reverse, 5'-GTCGGAGATTCGTAGCTGGA-3'; BUB1 mitotic checkpoint serine/threonine kinase (BUB1) forward, 5'-TGTCTT CCTCACCGATTCCT-3' and reverse, 5'-ACCACCCGAGCT CTGTCTTACTC-3'; GAPDH forward, 5'-AGGTCGGTG TGAACGGATTTG-3' and reverse, 5'-TGTAGACCATGT AGTTGAGGTCA-3'; complement C3 (C3) forward, 5'-CTC GCTTCGGCAGCACA-3' and reverse, 5'-AACGCTTCACGA ATTTGCGT-3'; kinesin family member 11 (KIF11) forward, 5'-TCCCTGAGACCCTAACTTGTGA-3' and reverse, 5'-AGT CTCAGGGTCCGAGGTATTC-3'; cyclin B2 (CCNB2) forward, 5'-GCACCGACCTCTTCAAGC-3' and reverse, 5'-CCATGAACTCCAGTCCTTCA3'; BUB1 mitotic checkpoint serine/threonine kinase B (Bublb) forward, 5'-AACCAG GATGGGTCACCATA-3' and reverse, 5'-ACTGAAACCCAA TGCACTCC-3'. GAPDH was used as the internal control. Relative gene expression levels were quantified using the $2^{-\Delta \Delta C q}$ method (21).

Venn diagram. A Venn diagram was drawn using jvenn (http://www.bioinformatics.com.cn/static/others/ jvenn/example.html) to determine the common DEGs between the datasets.

Statistical analysis. Values are expressed as the mean \pm standard deviation. Statistical analyses were performed using the Student's t-test using IBM SPSS version 21.0, software (IBM Corp.). $\mathrm{P}<0.05$ was considered to indicate a statistically significant difference.

\section{Results}

Identification of DEGs. The box plot of the log expression values for all genes in each group (control vs. IL-1 $\beta$-treated chondrocytes) prior to and after normalization were mapped (Fig. 1A). The median values of each sample were almost at a consistent level, suggesting that the data were eligible for further analysis.

The heatmap (Fig. 1B) and volcano plot (Fig. 1C) display the DEGs in IL-1 $\beta$-treated chondrocytes. Based on the cut-off criteria used for determination of the DEGs, a total of 844 DEGs, including 498 upregulated and 346 downregulated DEGs, were identified. The top 20 up- and downregulated DEGs are presented in Tables II and III, respectively.

The top 20 upregulated DEGs were as follows: Serum amyloid A2, C-X-C motif chemokine ligand 5, matrix metallopeptidase 3, C-X-C motif chemokine ligand 1, C-C motif chemokine ligand 7 , nitric oxide synthase 2 , matrix metallopeptidase 13 , vanin $1, \mathrm{C}-\mathrm{C}$ motif chemokine ligand 2 , haptoglobin,

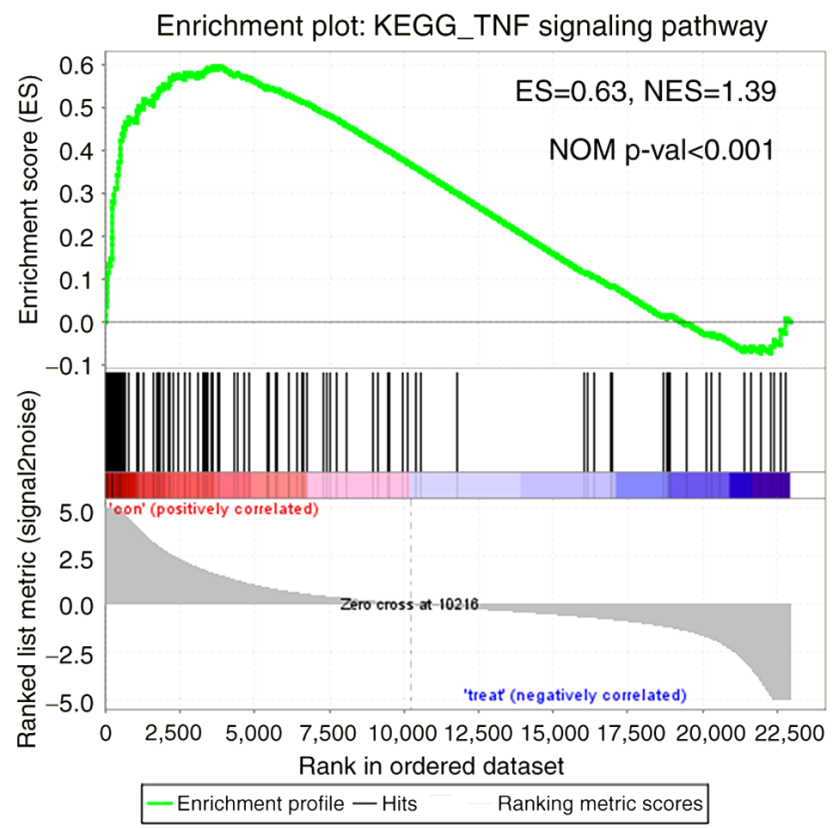

Figure 3. GSEA results presenting 'TNF signaling pathway' signatures enriched in IL-1 $\beta$-treated chondrocytes. GSEA, Gene Set Enrichment Analysis; ES, enrichment score; NES, normalized ES; NOM p-val, normalized P-value; KEGG, Kyoto Encyclopedia of Genes and Genomes; IL, interleukin.

serum amyloid A1, vanin 3, serpin family $\mathrm{G}$ member 1, SLIT and NTRK like family member 6, C-X-C motif chemokine ligand 3, serum amyloid A4, constitutive, NFKB inhibitor zeta, cholesterol 25-hydroxylase, adrenomedullin and solute carrier family 39 member 8 . The top 20 downregulated DEGs were Clq and TNF related 3, collagen type $X$ alpha 1 chain, sphingomyelin phosphodiesterase 3, fibroblast growth factor receptor 3, E2F transcription factor 8, solute carrier family 35 member G1, MAP7 domain containing 3, fibroblast growth factor 21, melanoma inhibitory activity 1, matrilin 3, microRNA (miR)-1983, noncompact myelin associated protein, C-type lectin domain family 3 member A, SHC binding and spindle associated 1, cytochrome b5 reductase 2, solute carrier family 38 member 3 , kinesin family member 11, family with sequence similarity 180 member A, myelin protein zero like 2 and miRNA 323.

GO term and KEGG pathway enrichment analysis of DEGs. GO enrichment analysis of DEGs using the Metascape online tool identified the enriched GO and KEGG terms, of which the top 20 were as follows: 'Inflammatory response', 'negative regulation of cell proliferation', 'ossification', 'taxis', 'blood vessel morphogenesis', 'extracellular structure organization', 'mitotic cell cycle process', 'TNF- $\alpha$ signaling pathway', 'muscle cell proliferation', 'positive regulation of cellular component movement', 'signal transduction by protein phosphorylation', 'leukocyte migration', 'epithelial cell proliferation', 'skeletal system development', 'cellular response to growth factor stimulus', 'negative regulation of cell differentiation', 'apoptotic signaling pathway', 'development of primary female sexual characteristics' and 'development growth and cell-cell adhesion' (Fig. 2). Detailed information about the GO and KEGG terms is presented in Table SI. GSEA was performed using the data profile of differentially expressed mRNAs and the most 

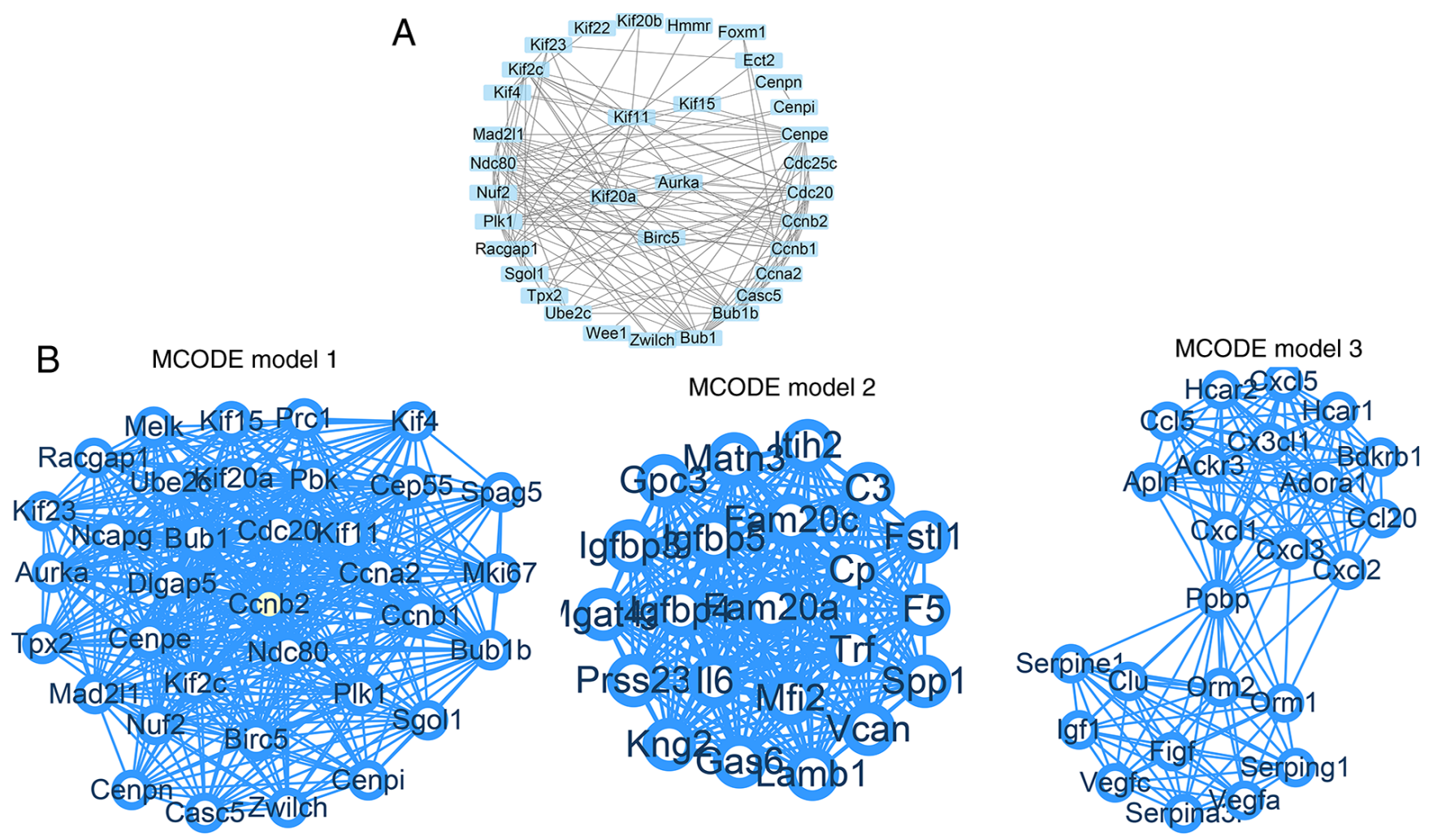

Figure 4. (A) Protein-protein interaction network of the DEGs. (B) Identification of a sub-network using Molecular Complex Detection in Cytoscape software. $\mathrm{P}<0.05$. DEGs, differentially expressed genes.

significantly enriched KEGG pathway was the TNF signaling pathway (Fig. 3).

PPI network and MCODE model in the interaction network. DEGs were entered into the STRING database to obtain the gene connection network, which contained 778 nodes and 1,641 edges. The average node degree was 4.22 and the average local clustering coefficient was 0.353 . The PPI enrichment P-value was $<1.0 \times 10^{-16}$ and was thus identified as statistically significant (Fig. 4). The remaining modules are shown in Fig. S1.

In order to improve the analysis of the PPI network, 14 modules were detected using the MCODE plugin. The top five modules are presented in Fig. 5.

Apoptosis and RT-qPCR results. To confirm that the cell model of OA was successfully established, the Annexin V-FITC/PI assay was performed. The results of the Annexin V-FITC/PI assay indicated that after treatment with IL-1 $\beta$, the apoptotic cells were significantly increased $\sim 3$-fold compared with those in the control group (7.5 vs. 23.5 ; $\mathrm{P}<0.05$; Fig. $6 \mathrm{~A}$ ). To verify the bioinformatics results, RT-qPCR was used to assess the top 10 hub genes in chondrocytes treated with IL-1b, including three upregulated genes (KNG2, $\mathrm{C} 3$ and BUB1B) and seven downregulated genes (CCNB1, CDC20, CCNA2, PIK1, BUB1, KIF11 and CCNB2). All of the results in the present study were consistent with the bioinformatics results ( $\mathrm{P}<0.05$; Fig. 6B).

The top 10 hub genes were also measured in cartilage samples from patients with $\mathrm{OA}$ and patients with normal cartilage. The in vivo PCR results were in accordance with the in vitro results, as the three upregulated genes (KNG2, $\mathrm{C} 3$ and

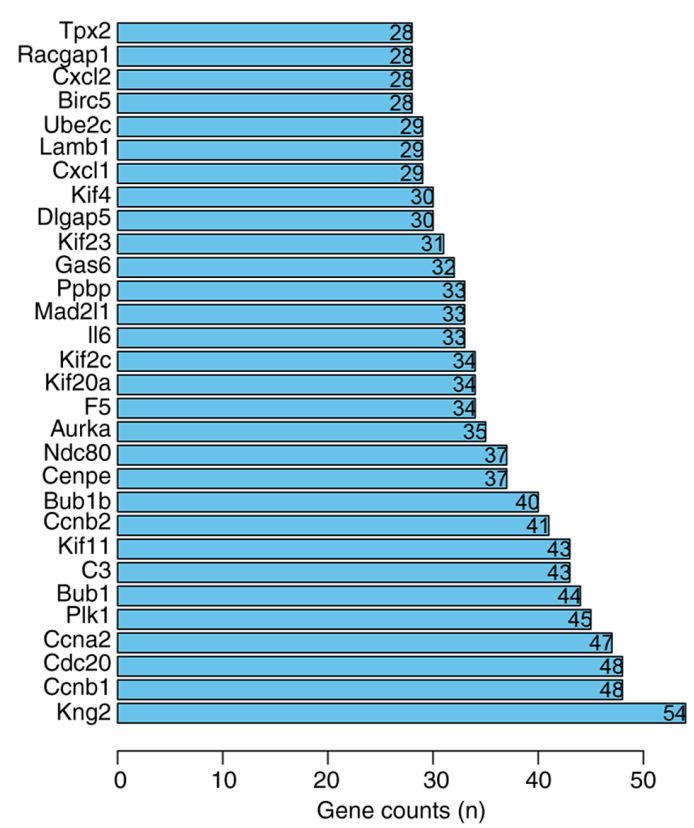

Figure 5. Top 30 core proteins of the protein-protein interaction network. The names of the genes are presented on the ordinate and the abscissa represents the number of gene connections. Tpx2, TPX2 microtubule nucleation factor; Racgap1, Rac GTPase activating protein 1; Cxcl, platelet factor 4; Birc5, baculoviral IAP repeat containing 5; Ube2c, ubiquitin conjugating enzyme E2 C; Lamb1, laminin subunit beta 1; Kif, kinesin family member; Dlgap5, DLG associated protein 5; Gas6, growth arrest specific 6; Ppbp, pro-platelet basic protein; Mad211, mitotic arrest deficient 2 like 1; F5, coagulation factor V; Aurka, aurora kinase A; Ndc80, NDC80 kinetochore complex component; Cenpe, centromere protein E; Bub1b, BUB1 mitotic checkpoint serine/ threonine kinase B; Ccnb2, cyclin B2; C3, complement C3; Bub1, BUB1 mitotic checkpoint serine/threonine kinase; Plk1, polo like kinase 1; Ccna2, cyclin A2; Cdc20, cell division cycle 20; Ccnb1, cyclin B1; Kng2, kininogen 2. 

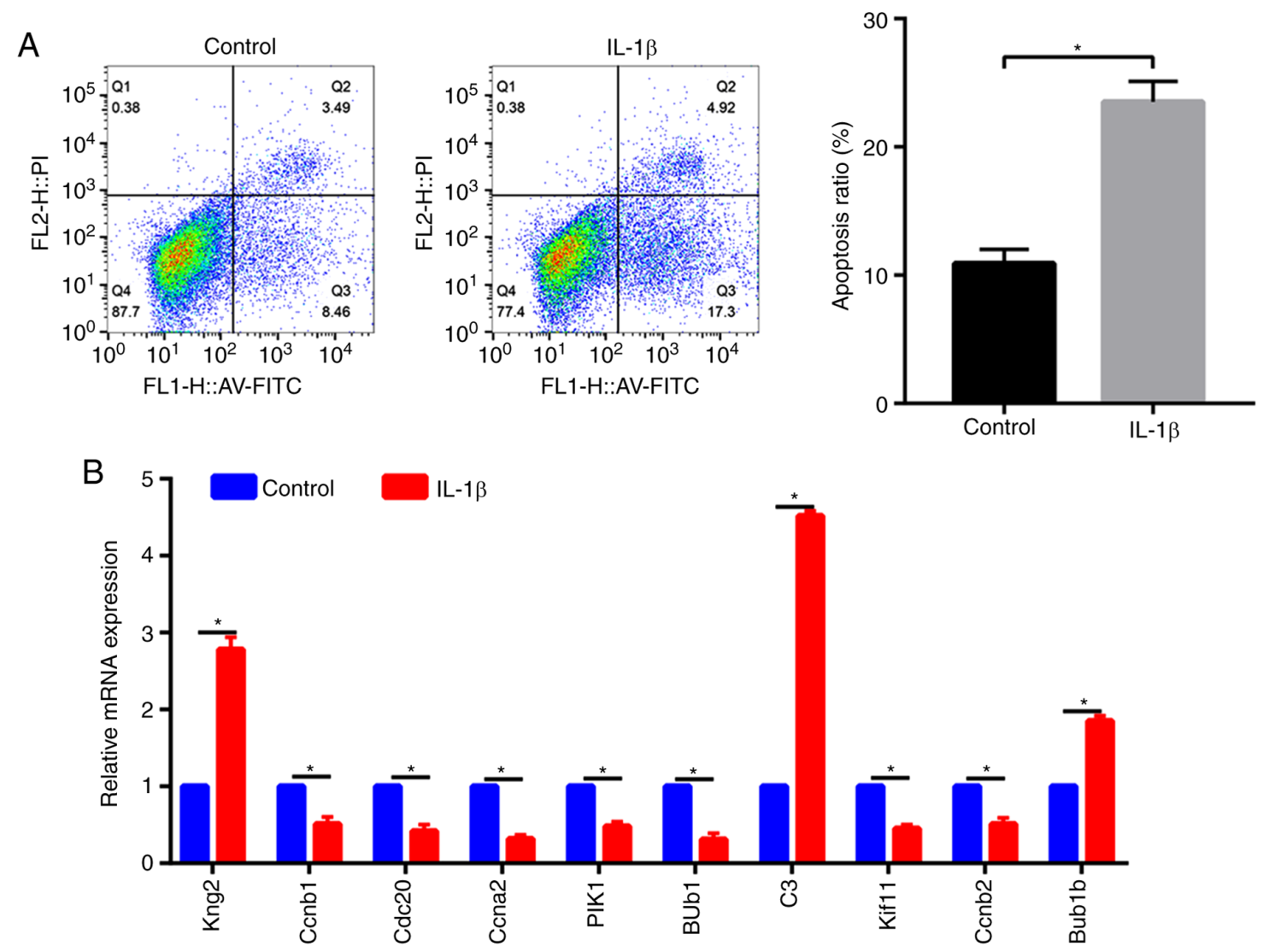

Figure. 6 (A) Effects of IL-1 $\beta$ on the induction of early apoptosis (AV-FITC staining) and late apoptosis (PI staining) as measured by flow cytometry. (B) Reverse transcription PCR analysis of the expression of the top 10 hub genes in IL-1 $\beta$-treated chondrocytes. "P<0.05. PI, propidium iodide; IL, interleukin; AV, Annexin V; Q, quadrant. Kng2, kininogen 2, Ccnb2, cyclin B2; Cdc20, cell division cycle 20; Ccna2, cyclin A2; Plk1, polo like kinase 1; Bub, BUB mitotic checkpoint serine/threonine kinase; C3, complement C3; Kif11, kinesin family member 11.

BUB1B) and seven downregulated genes (CCNB1, CDC20, CCNA2, PIK1, BUB1, KIF11 and CCNB2) were identified from OA cartilage and normal cartilage (Fig. 7).

A Venn diagram was then created to identify the overlapping genes between the GSE104793 and GSE74200 datasets. The 10 hub genes from the dataset GSE104793 were all identified from the dataset GSE74200 also (Fig. 8).

\section{Discussion}

OA is a disease with a high incidence rate worldwide, which eventually leads to joint dysfunction. Inflammatory factor-induced chondrocyte apoptosis leads to cartilage degradation and is considered to be key in the pathological development of OA. Among these inflammatory factors, cells challenged with IL-1 $\beta$ have been widely used to mimic arthritis in in vitro studies. Previous studies have indicated that apoptosis is significantly increased in IL-1 $\beta(10 \mathrm{ng} / \mathrm{ml})$-induced chondrocytes $(22,23)$. Bai et al (22) used different concentrations of IL-1 $\beta(0.5,1.0$, 2.5, 5.0, 10.0 and $20.0 \mathrm{ng} / \mathrm{ml}$ ) to injure chondrocytes and their results suggested that the optimal dose of IL- $1 \beta$ was $10 \mathrm{ng} / \mathrm{ml}$. Therefore, in the present study, an IL-1 $\beta$ concentration of $(10 \mathrm{ng} / \mathrm{ml})$ was selected for subsequent experiments.

Of note, the downstream genes induced following IL-1 $\beta$ stimulation to promote chondrocyte apoptosis have remained largely elusive, and their investigation was the purpose of the present study.
First, the mRNA expression profile data for IL-1 $\beta$-treated chondrocytes were obtained. By using the Limma package, a total of 844 DEGs were identified and the function of these DEGs was further analyzed. A major result was that DEGs following stimulation with IL-1 $\beta$ were mainly enriched in 'inflammatory response' (GO:0006954) and 'negative regulation of cell proliferation' (GO:0008285). IL-1 $\beta$, a member of the IL-1 family of cytokines, has a vital role in modulating the inflammatory response and is also involved in the proliferation, differentiation and apoptosis of multiple cell types, including chondrocytes (11), macrophages (24) and neuronal cells (25).

Another important finding was that IL-1 $\beta$-stimulated DEGs in chondrocytes were enriched in 'ossification'. It is already well known that endochondral ossification and $\mathrm{OA}$ are closely associated (26). Furthermore, anti-osteogenic reagents decrease the cartilage degeneration rate (27). Considering the GO functions of IL-1 $\beta$-stimulated DEGs in chondrocytes, further studies should focus on the association between ossification and chondrocyte apoptosis.

Considering the results of the KEGG pathway analysis, the present study linked the DEGs mainly with the TNF signaling pathway. Hamasaki et al (28) performed a study that focused on macrophages and cartilage fragments. The most significantly enriched terms were 'scavenger receptor activity' and 'TNF signaling'. TNF- $\alpha$ is a member of TNF signaling and is able to activate NF- $\kappa \mathrm{B}$ signaling to induce cartilage damage (29). Ren et al (30) performed a bioinformatics 

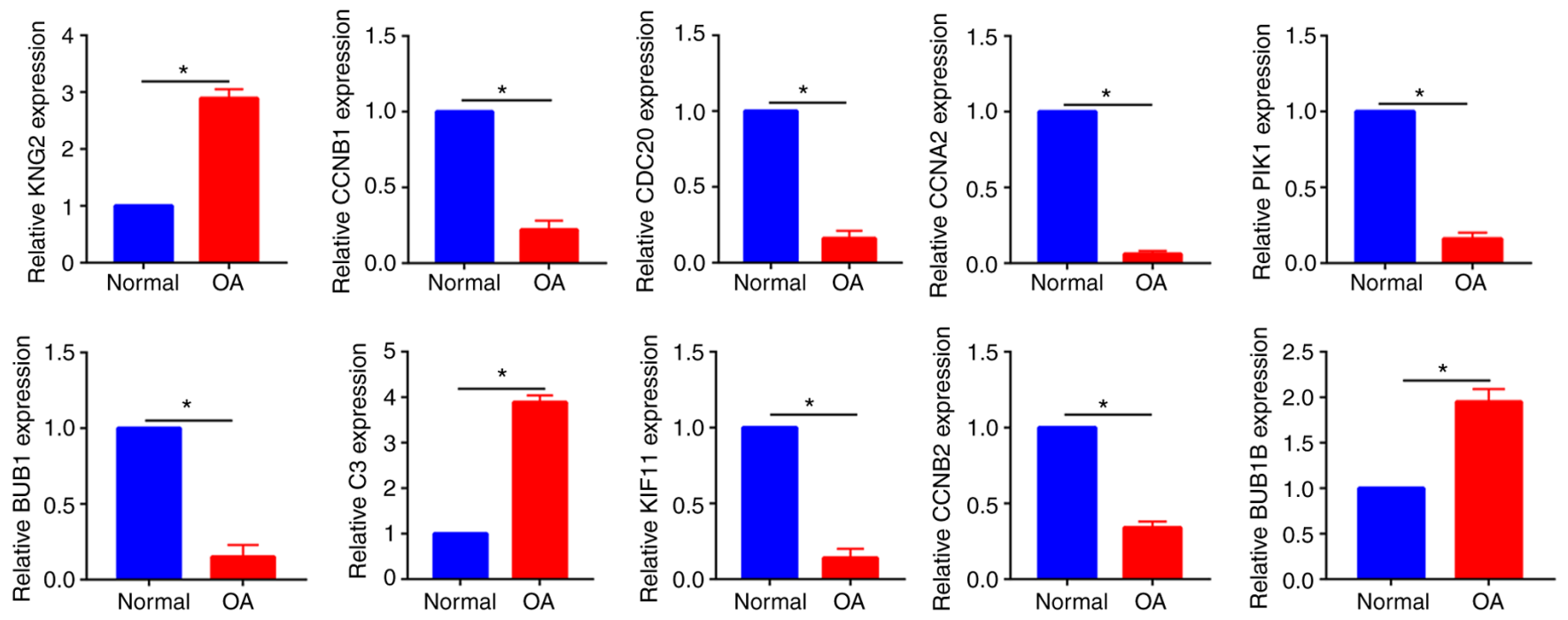

Figure 7. Reverse transcription PCR analysis of the expression of the top 10 hub genes in OA cartilage than normal cartilage. ${ }^{*}<0.05$. OA, osteoarthritis. Kng2, kininogen 2; Ccnb2, cyclin B2; Cdc20, cell division cycle 20; Ccna2, cyclin A2; Plk1, polo like kinase 1; Bub, BUB mitotic checkpoint serine/threonine kinase; C3, complement C3; Kif11, kinesin family member 11.

analysis that focused on the DEGs in knee OA in a rat model. The TNF signaling pathway was also investigated in an in vivo model. Thus, the detailed mechanisms underlying how the TNF signaling pathway induces OA progression are worthy of in-depth investigation.

The apoptotic signaling pathway was enriched by the DEGs. Tew et al (31) revealed that apoptosis and proliferation were two common biological processes during OA progression. Treatment with IL-1 $\beta$ also activated the apoptotic signaling pathway and thus, the inhibition of IL- $1 \beta$-induced chondrocyte apoptosis may delay OA progression.

In the present study, RT-qPCR analysis was performed in order to validate the results of the bioinformatics analysis. Treatment with IL-1 $\beta$ at $10 \mathrm{ng} / \mathrm{ml}$ for $24 \mathrm{~h}$ was used to induce an OA-like cell model. Apoptosis analysis was used to validate the effects of IL-1 $\beta$ on chondrocyte apoptosis. The top 10 hub genes were selected for further analysis. It was revealed that BUB1 was significantly downregulated in IL-1 $\beta$-treated chondrocytes. BUB1, BUB1B, CCNB1, CCNB2 and CCNA2 are known to participate in the mitotic cell cycle process (32). The most significant hub gene was KNG2, which is preferentially expressed in brown adipose tissue (33). Furthermore, KNG2 was regulated by cold stress, which suggested a role in thermoregulation (34). KNG2 mainly participates in the negative regulation of endopeptidase activity, signaling binding and positive regulation of cytosolic calcium ion concentration. These biological processes are all associated with cell apoptosis and signaling transduction (35). Another upregulated gene among the hub genes was C3. C3 was reported to inhibit RhoA GTPase activity and finally regulate Yes1-associated transcriptional regulator protein expression and its downstream target gene connective tissue growth factor to downregulate chondrocyte migration (36). Cyclin-dependent kinase (CDK)1 and BUB1B are two inflammation-associated critical signaling molecules. A previous study indicated that CDK1 and BUB1B were mainly involved in cell cycle regulation and cell movement (37). CCNB1 and CDC20 were identified as crucial genes associated with the pathogenesis and prognosis of pancreatic

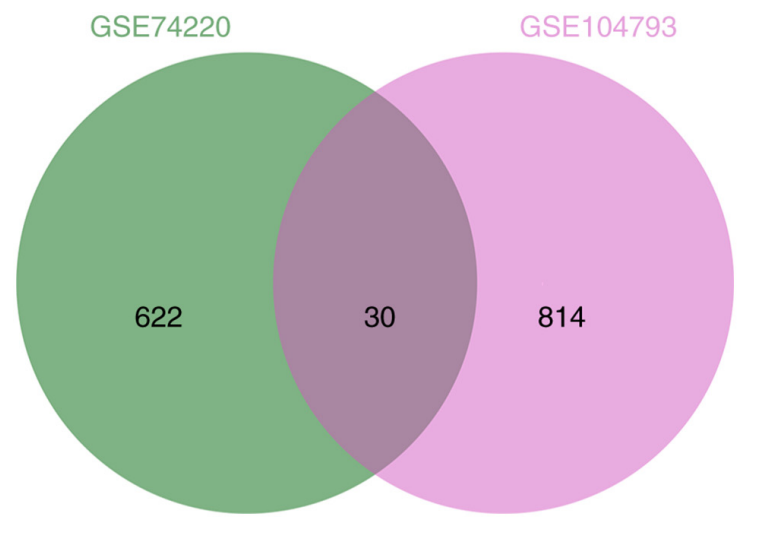

Figure 8. Common differentially expressed genes obtained from the GSE104793 and GSE74220 datasets using the Venn diagram web tool (http:// www.bioinformatics.com.cn/static/others/jvenn/example.html).

adenocarcinoma (38) and hepatocellular carcinoma $(39,40)$. CCNB1 and CDC20 were indicated to be associated with cell cycle and cell proliferation and thus have an important role in tumor progression (41). However, the effects of these genes on OA progression have remained elusive. In future studies, small interfering RNAs for KNG2 should be designed in order to investigate the role of the KNG2 gene in OA development.

The integrated bioinformatics analysis and experimental validation of the present study had several limitations that are worth mentioning. First, the results of the bioinformatics analysis were based on pre-set criteria; changing the cut-off criteria would ultimately affect the final results. Furthermore, the protein expression levels of these hub genes in each group were not assessed. Future studies should consider performing gene-modification-based therapy to treat OA in vivo and investigate the specific mechanisms of action.

In conclusion, in the present study, microarray data analysis for OA was first performed and 844 DEGs were identified, among which 498 DEGs were upregulated and 346 DEGs were downregulated. The identified DEGs may be involved 
in the inflammatory response, negative regulation of cell proliferation, ossification and TNF signaling. BUB1, BUB1B, CCNB1, CCNB2 andCCNA2 were hub genes, which were further validated by RT-qPCR. The functions of these DEGs require further verification using more in vivo and in vitro experiments.

\section{Acknowledgements}

Not applicable.

\section{Funding}

No funding was received.

\section{Availability of data and materials}

The datasets used and/or analyzed during the current study are available from the corresponding author on reasonable request.

\section{Authors' contributions}

FL and LP designed the study. YGM and WH performed the bioinformatic analysis. WBZ, MD and YML supervised the experiments. WBZ, MD and YML supervised the experiments and performed the in vitro experiments, including apoptotic analysis and real-time PCR. All authors read and approved the final version of the manuscript. FL and YML confirm the authenticity of all the raw data.

\section{Ethics approval and consent to participate}

The animal and human studies were approved by The Ethics Committee of Renmin Hospital of Wuhan University (animal approval no. 2019-YLS-0125) Wuhan, China. Written informed consent was obtained from all human subjects.

\section{Patient consent for publication}

Not applicable.

\section{Competing interests}

The authors declare that they have no competing interests.

\section{References}

1. Driban JB, Harkey MS, Barbe MF, Ward RJ, MacKay JW Davis JE, Lu B, Price LL, Eaton CB, Lo GH and McAlindon TE: Risk factors and the natural history of accelerated knee osteoarthritis: Anarrative review. BMC Musculoskelet Disord 21: 332, 2020 .

2. Kraus VB and Karsdal MA: Osteoarthritis: Current molecular biomarkers and the way forward. Calcif Tissue Int: May 4, 2020 (Epub ahead of print).

3. Huang W, Ong TY, Fu SC and Yung SH: Prevalence of patellofemoral joint osteoarthritis after anterior cruciate ligament injury and associated risk factors: A systematic review. J Orthop Translat 22: 14-25, 2019.

4. Chen AT, Shrestha S, Collins JE, Sullivan JK, Losina E and Katz JN: Estimating contextual effect in nonpharmacological therapies for pain in knee osteoarthritis: A systematic analytic review. Osteoarthritis Cartilage 28: 1154-1169, 2020.
5. Luo P, Xiong Z, Sun W, Shi L, Gao F and Li Z: How to Choose platelet-rich plasma or hyaluronic acid for the treatment of knee osteoarthritis in overweight or obese patients: A Meta-Analysis. Pain Res Manag 2020: 7587936, 2020.

6. Zhao Z, Ma JX and Ma XL: Different Intra-articular injections as therapy for hip osteoarthritis: A systematic review and network meta-analysis. Arthroscopy 36: 1452-1464.e2, 2020.

7. Honvo G, Leclercq V, Geerinck A, Thomas T, Veronese N, Charles A, Rabenda V, Beaudart C, Cooper C, Reginster JY and Bruyère O: Safety of topical non-steroidal anti-inflammatory drugs in osteoarthritis: Outcomes of a systematic review and meta-analysis. Pharmacol Rep 36 (Suppl 1): S45-S64, 2019.

8. Gregori D, Giacovelli G, Minto C, Barbetta B, Gualtieri F, Azzolina D, Vaghi P and Rovati LC: Association of pharmacological treatments with long-term pain control in patients with knee osteoarthritis: A systematic review and Meta-analysis. JAMA 320: 2564-2579, 2018.

9. Ahmad N, Ansari MY, Bano S and Haqqi TM: Imperatorin suppresses IL-1 $\beta$-induced iNOS expression via inhibiting ERK-MAPK/AP1 signaling in primary human OA chondrocytes. Int Immunopharmacol 85: 106612, 2020.

10. Dinarello CA: Blocking IL-1 in systemic inflammation. J Exp Med 201: 1355-1359, 2005.

11. Shen XF, Cheng Y, Dong QR and Zheng MQ: MicroRNA-675-3p regulates IL-1 $\beta$-stimulated human chondrocyte apoptosis and cartilage degradation by targeting GNG5. Biochem Biophys Res Commun 527: 458-465, 2020.

12. Shao J, Ding Z, Peng J, Zhou R, Li L, Qian Q and Chen Y: MiR-146a-5p promotes IL-1 $\beta$-induced chondrocyte apoptosis through the TRAF6-mediated NF-kB pathway. Inflamm Res 69: 619-630, 2020.

13. Jia Y, He W, Zhang H, He L, Wang Y, Zhang T, Peng J, Sun P and Qian Y: Morusin Ameliorates IL-1 $\beta$-Induced chondrocyte inflammation and osteoarthritis via NF- $\mathrm{KB}$ signal pathway. Drug Des Devel Ther 14: 1227-1240, 2020.

14. Wu Z, Irizarry RA, Gentleman R, Martinez-Murillo F and Spencer F: A model-based background adjustment for oligonucleotide expression arrays. J Am Statistical Association 99: 909-917, 2004.

15. R Core Team: R: A Language and Environment for Statistical Computing. R Foundation for Statistical Computing, Vienna, 2013. http://www.R-project.org/.

16. Zhou Y, Zhou B, Pache L, Chang M, Khodabakhshi AH, Tanaseichuk O, Benner $\mathrm{C}$ and Chanda SK: Metascape provides a biologist-oriented resource for the analysis of systems-level datasets. Nat Commun 10: 1523, 2019.

17. Kohl M, Wiese S and Warscheid B: Cytoscape: Software for visualization and analysis of biological networks. Methods Mol Biol 696: 291-303, 2011.

18. Bi W, Huang W, Whitworth DJ, Deng JM, Zhang Z, Behringer RR and de Crombrugghe B: Haploinsufficiency of Sox9 results in defective cartilage primordia and premature skeletal mineralization. Proc Natl Acad Sci USA 98: 6698-6703, 2001.

19. Johnson CI, Argyle DJ and Clements DN: In vitro models for the study of osteoarthritis. Vet J 209: 40-49, 2016.

20. Chen X, Wang R, Chen W, Lai L and Li Z: Decoy receptor-3 regulates inflammation and apoptosis via PI3K/AKT signaling pathway in coronary heart disease. Exp Ther Med 17: 2614-2622, 2019.

21. Zhao Z, Ma X, Ma J, Sun X, Li F and Lv J: Naringin enhances endothelial progenitor cell (EPC) proliferation and tube formation capacity through the CXCL12/CXCR4/PI3K/Akt signaling pathway. Chem Biol Interact 286: 45-51, 2018.

22. Bai M, Ge L, Chen H and Jin Q: Calcitonin protects rat chondrocytes from IL-1 $\beta$ injury via the Wnt/ $\beta$-catenin pathway. Exp Ther Med 18: 2079-2085, 2019

23. Li Z, Cheng J and Liu J: Baicalin protects human OA chondrocytes against IL-1 $\beta$-induced apoptosis and ECM degradation by activating autophagy via MiR-766-3p/AIFM1 axis. Drug Des Devel Ther 14: 2645-2655, 2020.

24. Fei Q, Ma H, Zou J, Wang W, Zhu L, Deng H, Meng M, Tan S, Zhang H, Xiao X, et al: Metformin protects against ischaemic myocardial injury by alleviating autophagy-ROS-NLRP3-mediated inflammatory response in macrophages. J Mol Cell Cardiol 145: 1-13, 2020.

25. Liu X, Chen Y, Wang H, Wei Y, Yuan Ye, Zhou Q, Fang F, Shi S, Jiang X, Dong Y and Li X: Microglia-derived IL-1 $\beta$ promoted neuronal apoptosis through ER stress-mediated signaling pathway PERK/eIF2 $\alpha /$ ATF4/CHOP upon arsenic exposure. J Hazard Mater, 417: 125997, 2021. 
26. Hosaka Y, Saito T, Sugita S, Hikata T, Kobayashi H, Fukai A Taniguchi $\mathrm{Y}$, Hirata M, Akiyama $\mathrm{H}$, Chung UI and Kawaguchi $\mathrm{H}$ : Notch signaling in chondrocytes modulates endochondral ossification and osteoarthritis development. Proc Natl Acad Sci USA 110: 1875-1880, 2013.

27. Hsiao HY, Cheng CM, Kao SW, Liu JW, Chang CS, Harhaus L and Huang JJ: The effect of bone inhibitors on periosteum-guided cartilage regeneration. Sci Rep 10: 8372, 2020.

28. Hamasaki M, Terkawi MA, Onodera T, Tian Y, Ebata T, Matsumae G, Alhasan H, Takahashi D and Iwasaki N: Transcriptional profiling of murine macrophages stimulated with cartilage fragments revealed a strategy for treatment of progressive osteoarthritis. Sci Rep 10: 7558, 2020.

29. Gao Y, Wang S, He L, Wang C and Yang L: Alpinetin protects chondrocytes and exhibits anti-inflammatory effects via the NF- $\kappa \mathrm{B} / \mathrm{ERK}$ pathway for alleviating osteoarthritis. Inflammation 43: 1742-1750, 2020.

30. Ren YM, Zhao X, Yang T, Duan YH, Sun YB, Zhao WJ and Tian MQ: Exploring the key genes and pathways of osteoarthritis in knee cartilage in a rat model using gene expression profiling. Yonsei Med J 59: 760-768, 2018.

31. Tew SR, Kwan AP, Hann A, Thomson BM and Archer CW: The reactions of articular cartilage to experimental wounding: Role of apoptosis. Arthritis Rheum 43: 215-225, 2000.

32. Sun J, Yan B, Yin W and Zhang X: Identification of genes associated with osteoarthritis by microarray analysis. Mol Med Rep 12: 5211-5216, 2015.

33. Peyrou M, Cereijo R, Quesada-López T, Campderrós L, Gavaldà-Navarro A, Liñares-Pose L, Kaschina E, Unger T, López M, Giralt M and Villarroya F: The kallikrein-kinin pathway as a mechanism for auto-control of brown adipose tissue activity. Nat Commun 11: 2132, 2020.

34. Rouhiainen A, Kulesskaya N, Mennesson M, Misiewicz Z, Sipilä T, Sokolowska E, Trontti K, Urpa L, McEntegart W, Saarnio S, et al: The bradykinin system in stress and anxiety in humans and mice. Sci Rep 9: 19437, 2019.
35. Sharma SK and Singh BR: Enhancement of the endopeptidase activity of purified botulinum neurotoxins $A$ and $E$ by an isolated component of the native neurotoxin associated proteins. Biochemistry 43: 4791-4798, 2004.

36. Jing X, Ye Y, Bao Y, Zhang J, Huang J, Wang R, Guo J and Guo F: Mechano-growth factor protects against mechanical overload induced damage and promotes migration of growth plate chondrocytes through RhoA/YAP pathway. Exp Cell Res 366: 81-91, 2018.

37. Kim EJ, Oh HY, Heo HS, Hong JE, Jung SJ, Lee KW, Park JH, Hur CG and Park JH: Biological features of core networks that result from a high-fat diet in hepatic and pulmonary tissues in mammary tumour-bearing, obesity-resistant mice. Br J Nutr 110: 241-255, 2013.

38. Shi LE, Shang X, Nie KC, Xu Q, Chen NB and Zhu ZZ: Identification of potential crucial genes associated with the pathogenesis and prognosis of pancreatic adenocarcinoma. Oncol Lett 20: 60, 2020.

39. Mi N, Cao J, Zhang J, Fu W, Huang C, Gao L, Yue P, Bai B, Lin Y, Meng W and Li X: Identification of hub genes involved in the occurrence and development of hepatocellular carcinoma via bioinformatics analysis. Oncol Lett 20: 1695-1708, 2020.

40. Meng Z, Wu J, Liu X, Zhou W, Ni M, Liu S, Guo S, Jia S and Zhang J: Identification of potential hub genes associated with the pathogenesis and prognosis of hepatocellular carcinoma via integrated bioinformatics analysis. J Int Med Res 48: 300060520910019,2020

41. Chen C, Guo Q, Song Y, Xu G and Liu L: SKA1/2/3 serves as a biomarker for poor prognosis in human lung adenocarcinoma. Transl Lung Cancer Res 9: 218-231, 2020.

This work is licensed under a Creative Commons Attribution-NonCommercial-NoDerivatives 4.0 International (CC BY-NC-ND 4.0) License. 